\title{
Estimation in Simple Step-Stress Model for the Marshall-Olkin Generalized Exponential Distribution under Type-I Censoring
}

\author{
F. L. Bagheri and Hamzeh Torabi*
}

Yazd University

\begin{abstract}
This paper considers the simple step-stress model from the Marshall-Olkin generalized exponential distribution when there is time constraint on the duration of the experiment. The maximum likelihood equations for estimating the parameters assuming a cumulative exposure model with lifetimes as the distributed Marshall-Olkin generalized exponential are derived. The likelihood equations do not lead to closed form expressions for the maximum likelihood estimators (MLEs), and they need to be solved by using an iterative procedure. We then evaluate the properties of MLEs through the mean squared error, relative absolute bias and relative error. We also derive confidence intervals for the parameters using asymptotic distributions of the MLEs and the parametric bootstrap methods. Finally, an example is presented to illustrate the discussed methods of asymptotic and bootstrap confidence intervals.
\end{abstract}

Keywords. Bootstrap method; cumulative exposure model; maximum likelihood estimation; Marshall-Olkin generalized exponential distribution; stepstress model; type-I censoring.

MSC 2010: 62F10, 62F12, 62F40, 62N01.

* Corresponding author 


\section{Introduction}

In most life-testing experiments, we can not continue the experiment until the last failure is observed based on cost, time and some other considerations. So, the experiment is usually terminated when either a pre-fixed censoring time $t$ arrives (Type-I censoring scheme) or when the $r$ th failure is observed (TypeII censoring scheme); see, for example, Harter and Balakrishnan (1996). In many situations, it may be difficult to collect data on life-time of a product under normal operating conditions as the product may have a high reliability under normal conditions. For this reason, accelerated life-testing (ALT) experiments can be used to force these products (systems or components) to fail more quickly than under normal operating conditions. Some key references in the area of accelerated testing include Nelson (1980, 1990), Meeker and Escobar (1998), and Bagdonavicius and Nikulin (2002).

A special class of the ALT is called the step-stress testing which allows the experimenter to choose one or more stress factors in a life-testing experiment. Stress factors can include humidity, temperature, vibration, voltage, load or any other factors that directly affect the life of the products. In such a life-testing experiment, $n$ identical units are placed on an initial stress level $s_{0}$ under a $m$-step-stress model, and only the successive failure times are recorded. The stress levels are changed to $s_{1}, \ldots, s_{m}$ at pre-fixed times $t_{1}, \ldots, t_{m}$, respectively. The most common model used to analyse these times-to- failure data is the cumulative exposure model.

We consider here a simple step-stress model with only two stress levels. This model has been studied extensively in the literature; Sedyakin (1966) introduced the cumulative exposure model in the simple step-stress case which has been further discussed and generalized by Bagdonavicius (1978) and Nelson (1980), while Miller and Nelson (1983) and Bai et al. (1989) discussed the determination of optimal time at which to change the stress level from $s_{0}$ and $s_{1}$. Xiong (1998), Xiong and Milliken (1999), and Balakrishnan et al. (2007) have all considered inferences for the step-stress model assuming exponential lifetimes based on complete, and Type-II censored samples. Balakrishnan et al. (2009) discussed exact inference for step-stress models under exponential distribution when the available data are Type-I censored. Balakrishnan and Iliopoulos (2010) established stochastic monotonicity of the MLEs of parameters in exponential simple step-stress models. Chen and Lio (2010) obtained the maximum likelihood estimation of the parameters in the generalized exponential distribution under progressive type-I interval 
censoring; Also Abdel-Hamid and AL-Hussaini (2009) estimated the parameters of the step-stress accelerated life tests for the exponentiated exponential distribution with Type-I censoring.

In the next section, we introduce a Marshall-Olkin generalized exponential distribution and then, we consider a simple step-stress model with two stress levels based on the Marshall-Olkin generalized exponential distribution when there is time constraint on the duration of the experiment. The model is described with details in Section 3. we derive maximum likelihood equations for estimating the parameters. But the likelihood equations do not lead to closed form expressions for the MLE, and they need to be solved by using an iterative procedure. In Section 4, asymptotic confidence intervals of the estimators are presented. In Section 5, asymptotic variance covariance matrix of the estimators are given. Simulation studies and properties of maximum likelihood estimators are given in Section 6. we discuss the bootstrap methods in Section 7. Finally, we state some results in Section 8.

\section{Marshall-Olkin Generalized Exponential Distri- bution}

The probability distribution function (PDF) of the Marshall-Olkin generalized exponential (MOGE) distribution with the parameters $p$ and $\theta$ is defined by

$$
f(x)=\frac{p e^{-\frac{x}{\theta}}}{\theta\left\{1-(1-p) e^{-\frac{x}{\theta}}\right\}^{2}}, \quad x>0,
$$

where $0<p \leqslant 1$ and $\theta>0$. The corresponding cumulative distribution function $(\mathrm{CDF})$ is

$$
F(x)=\frac{1-e^{-\frac{x}{\theta}}}{1-(1-p) e^{-\frac{x}{\theta}}}, \quad x>0 .
$$

If $p=1$, then the MOGE distribution reduces to the exponential distribution with the scale parameter $\theta$. This model was first proposed by Marshall and Olkin (1997) and extensively discussed by Alice and Jose (1999). Figure 1 illustrates the pdf of the MOGE distribution for some values of $p$ and $\theta$. 

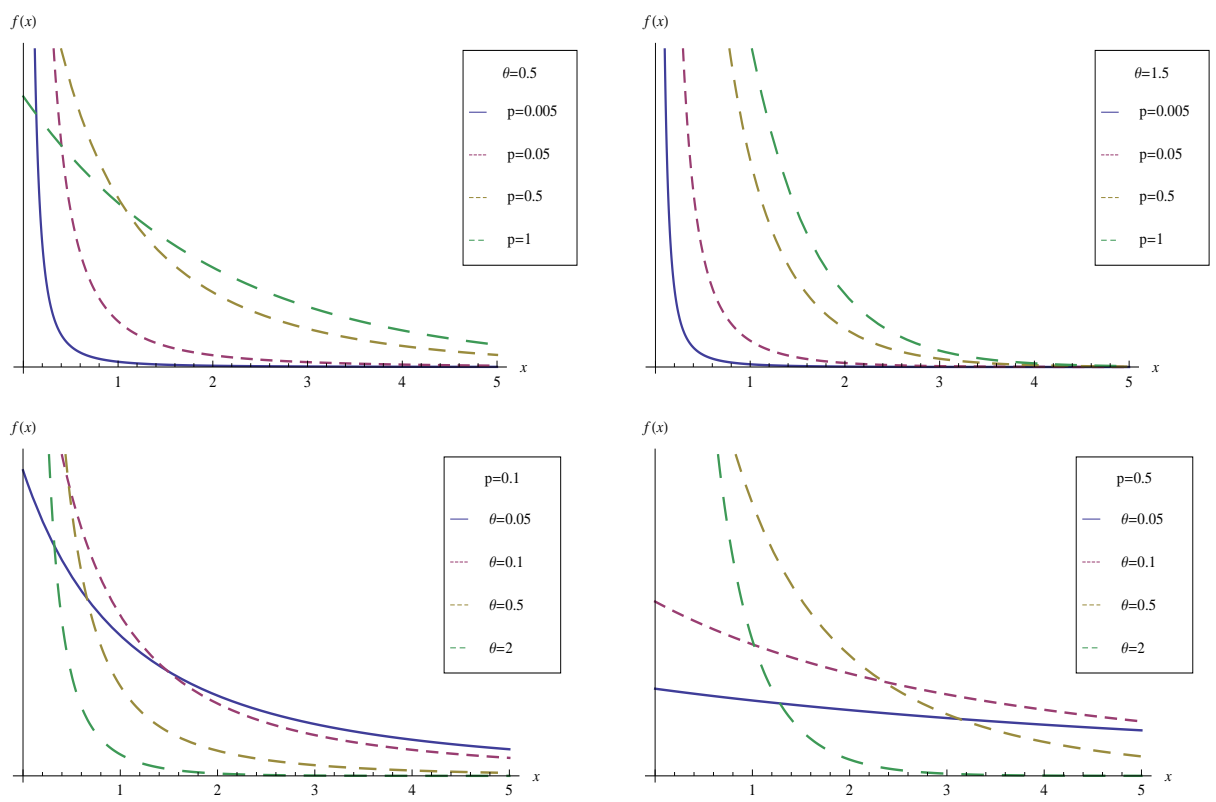

Figure 1. The pdf of the MOGE distribution for some values of $p$ and $\theta$.

\section{Step-stress Accelerated Life Tests under Cen- soring}

The term "Accelerated life test" applies to the type of study where failure times can be accelerated by applying higher "stress" to the component. This implies that the failure time is a function of the so called "stress factor" and higher stress may bring quicker failure. For example, some component may fail quicker at a higher temperature however, it may have a long life at lower temperatures. At low "stress" conditions, the time required may be too large for its reliability estimation which may be tested under higher stress factors terminating the experiment in a relatively shorter time, by this process failures which under normal conditions would occur only after a long testing can be observed quicker and the size of data can be increased without a large cost and long time. This type of reliability testing is called "Accelerated life testing". Accelerated life testing methods are also useful for obtaining information on the life of products or materials over a range 
of conditions, which are encountered in practice. Some information can be obtained by testing over the range of conditions of interest or over more severe conditions and then extrapolating the results over the range of interest. This type of test conditions are typically produced by testing units at high levels of temperature, voltage, pressure. vibration, cyclic rate, load etc. or some combination of them. Stress variables are used in engineering practice for many products and materials. In other fields similar problems arise when the relationship between variables could affect its life time. Therefore the models formulated are based on either past studies or theoretical development that could relate the distribution of failure time to stress or other variables. Such models are also useful in survival analysis where dependence of the life time of individuals on concomitant variables is analyzed (Sen, 1999); see for more details Nelsen $(1980,1990)$.

Suppose that the data come from a cumulative exposure model, and we consider a simple step-stress model with only two stress levels $s_{0}$ and $s_{1}$. The lifetime distributions at $s_{0}$ and $s_{1}$ are assumed to be the MOGE with parameters $\theta_{1}$ and $\theta_{2}$, respectively; and a common parameter $p$. The PDF and $\mathrm{CDF}$ are given by

$$
f_{k}\left(x ; p, \theta_{k}\right)=\frac{p e^{-\frac{x}{\theta_{k}}}}{\theta_{k}\left\{1-(1-p) e^{-\frac{x}{\theta_{k}}}\right\}^{2}},
$$

and

$$
F_{k}\left(x ; p, \theta_{k}\right)=\frac{1-e^{-\frac{x}{\theta_{k}}}}{1-(1-p) e^{-\frac{x}{\theta_{k}}}},
$$

respectively, where $x>0,0<p \leqslant 1, \theta_{k}>0, k=1,2$. We then have the cumulative exposure distribution (CED) $G(x)$ as

$$
G(x)= \begin{cases}G_{1}(x)=F_{1}\left(x ; \theta_{1}\right), & 0 \leqslant x<t_{1}, \\ G_{2}(x)=F_{2}\left(x-t_{1}+\frac{\theta_{2}}{\theta_{1}} t_{1} ; \theta_{2}\right), & t_{1} \leqslant x<\infty,\end{cases}
$$

where $F_{k}(\cdot)$ is given in (4). The corresponding PDF is

$$
g(x)= \begin{cases}g_{1}(x)=\frac{p e^{-\frac{x}{\theta_{1}}}}{\theta_{1}\left\{1-(1-p) e^{-\frac{x}{\theta_{1}}}\right\}^{2}}, & 0 \leqslant x<t_{1}, \\ g_{2}(x)=\frac{p e^{-\frac{x-t_{1}}{\theta_{2}}-\frac{t_{1}}{\theta_{1}}}}{\theta_{2}\left\{1-(1-p) e^{\left.-\frac{x-t_{1}}{\theta_{2}}-\frac{t_{1}}{\theta_{1}}\right\}^{2}}\right.} & t_{1} \leqslant x<\infty .\end{cases}
$$


Based on the Type-I censored data, we have $n$ identical units under an initial stress level $s_{0}$. The stress level is changed to $s_{1}$ at time $t_{1}$, and the life-testing experiment is terminated at time $t_{2}$, where $0<t_{1}<t_{2}<\infty$ are fixed in advance. Let $N_{1}$ be the number of units that fail before $t_{1}$, and $N_{2}$ be the number of units that fail before $t_{2}$ at stress level $s_{1}$, then, we will observe the following observations:

$$
x_{1: n}<x_{2: n}<\cdots<x_{N_{1}: n} \leqslant t_{1}<x_{N_{1}+1: n}<\cdots<x_{N_{1}+N_{2}: n} \leqslant t_{2}
$$

The likelihood of the observed failure times is then given by

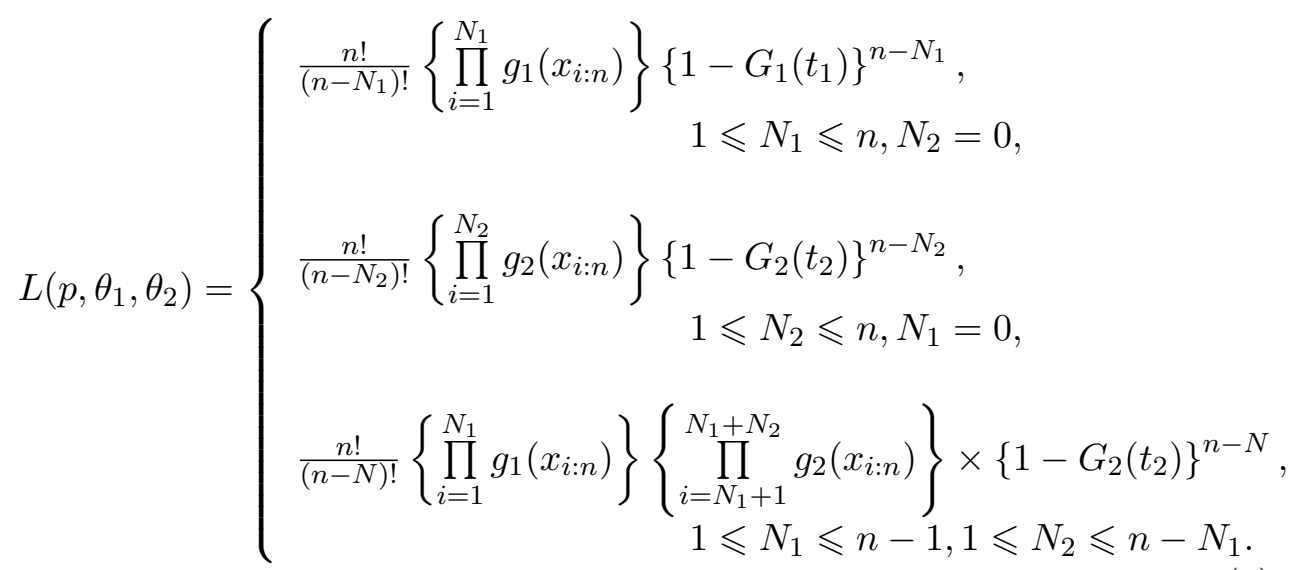

From (7) and (8), we observe the following:

1. If $N_{1}=0$ and $N_{2}=0$ in (7), the MLEs of $p, \theta_{1}$ and $\theta_{2}$ do not exist.

2. If $1 \leqslant N_{1} \leqslant n$ and $N_{2}=0$ in (7), the MLE of $\theta_{2}$ does not exist, since there are no failures observed after $t_{1}$.

3. If $N_{1}=0$ and $1 \leqslant N_{2} \leqslant n$ in (7), no failures could be observed before $t_{1}$. Therefore, the MLE of $\theta_{1}$ does not exist.

4. If $1 \leqslant N_{1} \leqslant n-1$ and $1 \leqslant N_{2} \leqslant n-N_{1}$ in (7), the MLEs of $p, \theta_{1}$ and $\theta_{2}$ exist. 
From (5) and (6), when $1 \leqslant N_{1} \leqslant n-1$ and $1 \leqslant N_{2} \leqslant n-N_{1}$ the loglikelihood function is given by

$$
\begin{aligned}
l\left(p, \theta_{1}, \theta_{2}\right)= & \text { const }+n \log (p)-N_{1} \log \left(\theta_{1}\right)-N_{2} \log \left(\theta_{2}\right)-\frac{1}{\theta_{1}} \sum_{i=1}^{N_{1}} x_{i: n} \\
& -2 \sum_{i=1}^{N_{1}} \log \left\{1-(1-p) e^{-\frac{x_{i: n}}{\theta_{1}}}\right\}-\frac{1}{\theta_{2}} \sum_{i=N_{1}+1}^{N_{1}+N_{2}}\left(x_{i: n}-t_{1}+\frac{\theta_{2}}{\theta_{1}} t_{1}\right) \\
& -2 \sum_{i=N_{1}+1}^{N_{1}+N_{2}} \log \left\{1-(1-p) e^{-\frac{x_{i: n}-t_{1}}{\theta_{2}}-\frac{t_{1}}{\theta_{1}}}\right\}-\left(n-N_{1}-N_{2}\right) \frac{t_{2}-t_{1}}{\theta_{2}} \\
& -\left(n-N_{1}-N_{2}\right) \frac{t_{1}}{\theta_{1}}-\left(n-N_{1}-N_{2}\right) \log \left\{1-(1-p) e^{-\frac{t_{2}-t_{1}}{\theta_{2}}-\frac{t_{1}}{\theta_{1}}}\right\}
\end{aligned}
$$

Our objective now is to determine the maximum likelihood estimates (MLE) of the parameters $p, \theta_{1}$ and $\theta_{2}$, based on the observed failure times. These estimates have to be viewed as conditional MLE because we are working under the condition that $1 \leqslant N_{1} \leqslant n-1$ and $1 \leqslant N_{2} \leqslant n-N_{1}$.

The log-likelihood function can be written as

$$
\begin{aligned}
& \frac{\partial l}{\partial p}=\frac{n}{p}-2 \sum_{i=1}^{N_{1}} \frac{e^{-\frac{x_{i: n}}{\theta_{1}}}}{1-(1-p) e^{-\frac{x_{i: n}}{\theta_{1}}}}-2 \sum_{i=N_{1}+1}^{N_{1}+N_{2}} \frac{e^{-\frac{x_{i: n}-t_{1}}{\theta_{2}}-\frac{t_{1}}{\theta_{1}}}}{1-(1-p) e^{-\frac{x_{i: n}-t_{1}}{\theta_{2}}-\frac{t_{1}}{\theta_{1}}}} \\
& -\left(n-N_{1}-N_{2}\right) \frac{e^{-\frac{t_{2}-t_{1}}{\theta_{2}}-\frac{t_{1}}{\theta_{1}}}}{1-(1-p) e^{-\frac{t_{2}-t_{1}}{\theta_{2}}-\frac{t_{1}}{\theta_{1}}}} \\
& \frac{\partial l}{\partial \theta_{1}}=-\frac{N_{1}}{\theta_{1}}+\frac{1}{\theta_{1}^{2}} \sum_{i=1}^{N_{1}} x_{i: n}+\frac{2}{\theta_{1}^{2}} \sum_{i=1}^{N_{1}} \frac{(1-p) x_{i: n} e^{-\frac{x_{i: n}}{\theta_{1}}}}{1-(1-p) e^{-\frac{x_{i: n}}{\theta_{1}}}}+\frac{N_{2} t_{1}}{\theta_{1}^{2}} \\
& +\frac{2}{\theta_{1}^{2}} \sum_{i=N_{1}+1}^{N_{1}+N_{2}} \frac{(1-p) t_{1} e^{-\frac{x_{i: n}-t_{1}}{\theta_{2}}-\frac{t_{1}}{\theta_{1}}}}{1-(1-p) e^{-\frac{x_{i: n}-t_{1}}{\theta_{2}}-\frac{t_{1}}{\theta_{1}}}}+\left(n-N_{1}-N_{2}\right) \frac{t_{1}}{\theta_{1}^{2}} \\
& +\frac{n-N_{1}-N_{2}}{\theta_{1}^{2}} \cdot \frac{(1-p) t_{1} e^{-\frac{t_{2}-t_{1}}{\theta_{2}}-\frac{t_{1}}{\theta_{1}}}}{1-(1-p) e^{-\frac{t_{2}-t_{1}}{\theta_{2}}-\frac{t_{1}}{\theta_{1}}}}
\end{aligned}
$$




$$
\begin{aligned}
\frac{\partial l}{\partial \theta_{2}}= & -\frac{N_{2}}{\theta_{2}}+\frac{1}{\theta_{2}^{2}} \sum_{i=N_{1}+1}^{N_{1}+N_{2}}\left(x_{i: n}-t_{1}\right)+\frac{2}{\theta_{2}^{2}} \sum_{i=N_{1}+1}^{N_{1}+N_{2}} \frac{(1-p)\left(x_{i: n}-t_{1}\right) e^{-\frac{x_{i: n}-t_{1}}{\theta_{2}}-\frac{t_{1}}{\theta_{1}}}}{1-(1-p) e^{-\frac{x_{i: n}-t_{1}}{\theta_{2}}-\frac{t_{1}}{\theta_{1}}}} \\
& +\left(n-N_{1}-N_{2}\right) \frac{t_{2}-t_{1}}{\theta_{2}^{2}}+\frac{n-N_{1}-N_{2}}{\theta_{2}^{2}} \cdot \frac{(1-p)\left(t_{2}-t_{1}\right) e^{-\frac{t_{2}-t_{1}}{\theta_{2}}-\frac{t_{1}}{\theta_{1}}}}{1-(1-p) e^{-\frac{t_{2}-t_{1}}{\theta_{2}}-\frac{t_{1}}{\theta_{1}}}}
\end{aligned}
$$

Numerical methods are applied for simultaneously solving the nonlinear equations to obtain parameters. The required numerical evaluations were implemented using the $R$ Software through the package (stats 4), command mle with the L-BFGS-B method.

\section{Interval Estimates}

Because the MLE of the model parameters are not in closed form expressions, it is not possible to derive their distributions, and therefore the corresponding exact confidence intervals (CI). Hence, we will discuss here the asymptotic confidence intervals.

For large sample size $n$, the derivation of the asymptotic confidence intervals (ACI) for the parameters $p, \theta_{1}$ and $\theta_{2}$ will be based on the pivotal quantities $(\hat{p}-E(\hat{p})) / \sqrt{V(\hat{p})},\left(\hat{\theta_{1}}-E\left(\hat{\theta_{1}}\right)\right) / \sqrt{V\left(\hat{\theta_{1}}\right)}$ and $\left(\hat{\theta_{2}}-E\left(\hat{\theta_{2}}\right)\right) / \sqrt{V\left(\hat{\theta_{2}}\right)}$, respectively. The maximum likelihood estimates, under appropriate regularity conditions (A1-A6 conditions stated in Casella and Berger (2002), Page 516) are consistent and asymptotically normally distributed. Therefore, we end up with the asymptotic two-sided $100(1-\alpha) \%$ CI of the form $\hat{p} \pm z_{\alpha / 2} \sqrt{V(\hat{p})}$, $\hat{\theta_{1}} \pm z_{\alpha / 2} \sqrt{V\left(\hat{\theta_{1}}\right)}$ and $\hat{\theta_{2}} \pm z_{\alpha / 2} \sqrt{V\left(\hat{\theta_{2}}\right)}$, where $z_{p}$ is the $p$-th upper percentile of the standard normal distribution. Here, $V(\hat{p}), V\left(\hat{\theta_{1}}\right)$ and $V\left(\hat{\theta_{2}}\right)$ are the diagonal elements of the inverse of the observed Fisher information matrix presented in next section. 


\section{Asymptotic Variances and Covariances of Esti- mates}

The asymptotic variances and covariances of maximum likelihood estimators are given by the elements of the inverse of the Fisher information matrix

$$
I_{i j}(\boldsymbol{\psi})=E\left\{-\partial^{2} l / \partial \psi_{i} \partial \psi_{j}\right\}, \quad i, j=1,2,3
$$

where $\boldsymbol{\psi}=\left(\psi_{1}, \psi_{2}, \psi_{3}\right), \psi_{1}=p, \psi_{2}=\theta_{1}$ and $\psi_{3}=\theta_{2}$.

Unfortunately, the exact mathematical expressions for the above expectations are very difficult to obtain. Therefore, the observed Fisher information matrix is given by $I_{i j}(\boldsymbol{\psi})=\left\{-\partial^{2} l / \partial \psi_{i} \partial \psi_{j}\right\}$, which is obtained by dropping the expectation operator $E$; for more details, see Cohen (1965).

The second partial derivatives of the maximum likelihood function are given as the following:

$$
\begin{aligned}
\frac{\partial^{2} l}{\partial p^{2}}=- & \frac{n}{p^{2}}+2 \sum_{i=1}^{N_{1}} \frac{e^{\frac{-2 x_{i: n}}{\theta_{1}}}}{\left\{1-(1-p) e^{-\frac{x_{i: n}}{\theta_{1}}}\right\}^{2}} \\
& +2 \sum_{i=N_{1}+1}^{N_{1}+N_{2}} \frac{\left(e^{-\frac{x_{i: n}-t_{1}}{\theta_{2}}-\frac{t_{1}}{\theta_{1}}}\right)^{2}}{\left\{1-(1-p) e^{-\frac{x_{i: n}-t_{1}}{\theta_{2}}-\frac{t_{1}}{\theta_{1}}}\right\}^{2}} \\
& +\left(n-N_{1}-N_{2}\right) \frac{\left(e^{-\frac{t_{2}-t_{1}}{\theta_{2}}-\frac{t_{1}}{\theta_{1}}}\right)^{2}}{\left\{1-(1-p) e^{-\frac{t_{2}-t_{1}}{\theta_{2}}-\frac{t_{1}}{\theta_{1}}}\right\}^{2}}
\end{aligned}
$$




$$
\begin{aligned}
& \frac{\partial^{2} l}{\partial p \partial \theta_{1}}=-\frac{2}{\theta_{1}^{2}} \sum_{i=1}^{N_{1}} \frac{x_{i: n} e^{-\frac{x_{i: n}}{\theta_{1}}}}{\left\{1-(1-p) e^{-\frac{x_{i: n}}{\theta_{1}}}\right\}^{2}} \\
& -\frac{2 t_{1}}{\theta_{1}^{2}} \sum_{i=N_{1}+1}^{N_{1}+N_{2}} \frac{e^{-\frac{x_{i: n}-t_{1}}{\theta_{2}}-\frac{t_{1}}{\theta_{1}}}}{\left\{1-(1-p) e^{-\frac{x_{i: n}-t_{1}}{\theta_{2}}-\frac{t_{1}}{\theta_{1}}}\right\}^{2}} \\
& -\frac{\left(n-N_{1}-N_{2}\right) t_{1}}{\theta_{1}^{2}} \cdot \frac{e^{-\frac{t_{2}-t_{1}}{\theta_{2}}-\frac{t_{1}}{\theta_{1}}}}{\left\{1-(1-p) e^{-\frac{t_{2}-t_{1}}{\theta_{2}}-\frac{t_{1}}{\theta_{1}}}\right\}^{2}} \\
& \frac{\partial^{2} l}{\partial p \partial \theta_{2}}=-\frac{2}{\theta_{2}^{2}} \sum_{i=N_{1}+1}^{N_{1}+N_{2}} \frac{\left(x_{i: n}-t_{1}\right) e^{-\frac{x_{i: n}-t_{1}}{\theta_{2}}-\frac{t_{1}}{\theta_{1}}}}{\left\{1-(1-p) e^{-\frac{x_{i: n}-t_{1}}{\theta_{2}}-\frac{t_{1}}{\theta_{1}}}\right\}^{2}} \\
& -\left(n-N_{1}-N_{2}\right) \frac{t_{2}-t_{1}}{\theta_{2}^{2}} \times \frac{e^{-\frac{t_{2}-t_{1}}{\theta_{2}}-\frac{t_{1}}{\theta_{1}}}}{\left\{1-(1-p) e^{-\frac{t_{2}-t_{1}}{\theta_{2}}-\frac{t_{1}}{\theta_{1}}}\right\}^{2}} \\
& \frac{\partial^{2} l}{\partial \theta_{1}^{2}}=\frac{N_{1}}{\theta_{1}^{2}}-\frac{2}{\theta_{1}^{3}} \sum_{i=1}^{N_{1}} x_{i: n}-\frac{4}{\theta_{1}^{3}} \sum_{i=1}^{N_{1}} \frac{(1-p) x_{i: n} e^{-\frac{x_{i: n}}{\theta_{1}}}}{1-(1-p) e^{-\frac{x_{i: n}}{\theta_{1}}}} \\
& +\frac{2}{\theta_{1}^{4}} \sum_{i=1}^{N_{1}} \frac{(1-p) x_{i: n}^{2} e^{-\frac{x_{i: n}}{\theta_{1}}}}{\left\{1-(1-p) e^{-\frac{x_{i: n}}{\theta_{1}}}\right\}^{2}} \\
& -\frac{2 N_{2} t_{1}}{\theta_{1}^{3}}-\frac{4 t_{1}}{\theta_{1}^{3}} \sum_{i=N_{1}+1}^{N_{1}+N_{2}} \frac{(1-p) e^{-\frac{x_{i: n}-t_{1}}{\theta_{2}}-\frac{t_{1}}{\theta_{1}}}}{1-(1-p) e^{-\frac{x_{i: n}-t_{1}}{\theta_{2}}-\frac{t_{1}}{\theta_{1}}}} \\
& +\frac{2}{\theta_{1}^{4}} \sum_{i=N_{1}+1}^{N_{1}+N_{2}} \frac{(1-p) t_{1}^{2} e^{-\frac{x_{i: n}-t_{1}}{\theta_{2}}-\frac{t_{1}}{\theta_{1}}}}{\left\{1-(1-p) e^{-\frac{x_{i: n}-t_{1}}{\theta_{2}}-\frac{t_{1}}{\theta_{1}}}\right\}^{2}}-\left(n-N_{1}-N_{2}\right) \frac{2 t_{1}}{\theta_{1}^{3}} \\
& -\frac{2\left(n-N_{1}-N_{2}\right)}{\theta_{1}^{3}} \cdot \frac{(1-p) t_{1} e^{-\frac{t_{2}-t_{1}}{\theta_{2}}-\frac{t_{1}}{\theta_{1}}}}{1-(1-p) e^{-\frac{t_{2}-t_{1}}{\theta_{2}}-\frac{t_{1}}{\theta_{1}}}}
\end{aligned}
$$




$$
\begin{aligned}
& +\frac{n-N_{1}-N_{2}}{\theta_{1}^{4}} \cdot \frac{(1-p) t_{1}^{2} e^{-\frac{t_{2}-t_{1}}{\theta_{2}}-\frac{t_{1}}{\theta_{1}}}}{\left\{1-(1-p) e^{-\frac{t_{2}-t_{1}}{\theta_{2}}-\frac{t_{1}}{\theta_{1}}}\right\}^{2}} \\
& \frac{\partial^{2} l}{\partial \theta_{1} \partial \theta_{2}}=\frac{2 t_{1}}{\theta_{1}^{2} \theta_{2}^{2}} \sum_{i=N_{1}+1}^{N_{1}+N_{2}} \frac{(1-p)\left(x_{i: n}-t_{1}\right) e^{-\frac{x_{i: n}-t_{1}}{\theta_{2}}-\frac{t_{1}}{\theta_{1}}}}{\left\{1-(1-p) e^{-\frac{x_{i: n}-t_{1}}{\theta_{2}}-\frac{t_{1}}{\theta_{1}}}\right\}^{2}} \\
& +\frac{n-N_{1}-N_{2}}{\theta_{1}^{2} \theta_{2}^{2}} \cdot \frac{(1-p) t_{1}\left(t_{2}-t_{1}\right) e^{-\frac{t_{2}-t_{1}}{\theta_{2}}-\frac{t_{1}}{\theta_{1}}}}{\left\{1-(1-p) e^{-\frac{t_{2}-t_{1}}{\theta_{2}}-\frac{t_{1}}{\theta_{1}}}\right\}^{2}} \\
& \frac{\partial^{2} l}{\partial \theta_{2}^{2}}=\frac{N_{2}}{\theta_{2}^{2}}-\frac{2}{\theta_{2}^{3}} \sum_{i=N_{1}+1}^{N_{1}+N_{2}}\left(x_{i: n}-t_{1}\right) \\
& -\frac{4}{\theta_{2}^{3}} \sum_{i=N_{1}+1}^{N_{1}+N_{2}} \frac{(1-p)\left(x_{i: n}-t_{1}\right) e^{-\frac{x_{i: n}-t_{1}}{\theta_{2}}-\frac{t_{1}}{\theta}} 1}{1-(1-p) e^{-\frac{x_{i: n}-t_{1}}{\theta_{2}}-\frac{t_{1}}{\theta_{1}}}} \\
& +\frac{2}{\theta_{2}^{4}} \sum_{i=N_{1}+1}^{N_{1}+N_{2}} \frac{(1-p)\left(x_{i: n}-t_{1}\right) e^{-\frac{x_{i: n}-t_{1}}{\theta_{2}}-\frac{t_{1}}{\theta_{1}}}}{\left\{1-(1-p) e^{-\frac{x_{i: n}-t_{1}}{\theta_{2}}-\frac{t_{1}}{\theta_{1}}}\right\}^{2}} \\
& -2\left(n-N_{1}-N_{2}\right) \frac{t_{2}-t_{1}}{\theta_{2}^{3}} \\
& -\left(n-N_{1}-N_{2}\right) \frac{2}{\theta_{2}^{3}} \cdot \frac{(1-p)\left(t_{2}-t_{1}\right) e^{-\frac{t_{2}-t_{1}}{\theta_{2}}-\frac{t_{1}}{\theta_{1}}}}{\left\{1-(1-p) e^{-\frac{t_{2}-t_{1}}{\theta_{2}}-\frac{t_{1}}{\theta_{1}}}\right\}^{2}} \\
& +\left(n-N_{1}-N_{2}\right) \frac{\left(t_{2}-t_{1}\right)^{2}}{\theta_{2}^{4}} \cdot \frac{(1-p) e^{-\frac{t_{2}-t_{1}}{\theta_{2}}-\frac{t_{1}}{\theta_{1}}}}{\left\{1-(1-p) e^{-\frac{t_{2}-t_{1}}{\theta_{2}}-\frac{t_{1}}{\theta_{1}}}\right\}^{2}}
\end{aligned}
$$

Consequently, the maximum likelihood estimators of $p, \theta_{1}$ and $\theta_{2}$ have an asymptotic variance covariance matrix defined by inverting the Fisher information matrix and then substituting $p, \theta_{1}$ and $\theta_{2}$ by $\hat{p}, \hat{\theta}_{1}$ and $\hat{\theta}_{2}$. 


\section{Simulation Study}

In order to obtain the MLEs of parameters and study the properties of their estimates through the mean squared errors (MSE), relative absolute biases (RAB) and relative errors (RE), we describe the algorithm to obtain the Type-I censored sample. A simulation study is performed according to the following steps:

For given values of $t_{1}, t_{2}$ and the parameters $p, \theta_{1}$ and $\theta_{2}$,

Step 1. we generate a random sample of size $n$ from Uniform $(0,1)$ distribution, and obtain the order statistics $\left(U_{1: n}, \ldots, U_{n: n}\right)$.

Step 2. Find $N_{1}$ such that

$$
\begin{aligned}
& U_{N_{1}: n}<P\left(X \leqslant t_{1}\right)=G_{1}\left(t_{1}\right) \leqslant U_{N_{1}+1: n} \\
& \Leftrightarrow U_{N_{1}: n}<\frac{e^{-\frac{t_{1}}{\theta_{1}}}}{1-(1-p) e^{-\frac{t_{1}}{\theta_{1}}}} \leqslant U_{N_{1}+1: n} .
\end{aligned}
$$

The values $x_{1: n}, x_{2: n}, \ldots, x_{N_{1}: n}$ construct a simulated random sample from the MOGE distribution in stress level $s_{0}$, since using the probability integral transformation theorem, the solution of the equation $U_{i: n}=G_{1}\left(x_{i: n}\right)$ with respect to $x_{i: n}, 1 \leqslant i \leqslant N_{1}$, is a simulated value from the distribution with the CDF $G_{1}(\cdot)$. But

$$
\begin{aligned}
& U_{i: n}=G_{1}\left(x_{i: n}\right) \Leftrightarrow U_{i: n}=\frac{e^{-\frac{x_{i: n}}{\theta_{1}}}}{1-(1-p) e^{-\frac{x_{i: n}}{\theta_{1}}}} \\
& \Leftrightarrow x_{i: n}=\theta_{1} \log \frac{1-(1-p) U_{i: n}}{1-U_{i: n}}, \quad i=1, \ldots, N_{1},
\end{aligned}
$$

Note that $G_{1}(\cdot)$ is an one-to-one function, so its inverse function, $x_{i: n}$ is the unique solution of the equation.

Step 3. Next, we generate a random sample of size $m=n-N_{1}$ from Uniform $(0,1)$ distribution, and obtain the order statistics $\left(V_{1: m}, \ldots, V_{m: m}\right)$. 
Step 4. Find $N_{2}$ such that

$$
\begin{aligned}
& V_{N_{2}: m}<P\left(X \leqslant t_{2} \mid X \geqslant t_{1}\right)=\frac{G_{2}\left(t_{2}\right)-G_{1}\left(t_{1}\right)}{1-G_{1}\left(t_{1}\right)} \leqslant V_{N_{2}+1: m} \\
& \Longleftrightarrow V_{N_{2}: m}<\frac{1-e^{-\frac{t_{2}-t_{1}}{\theta_{2}}}}{1-(1-p) e^{-\frac{t_{2}-t_{1}}{\theta_{2}}-\frac{t_{1}}{\theta_{1}}}} \leqslant V_{N_{2}+1: m} .
\end{aligned}
$$

The values $x_{N_{1}+1: n}, \ldots, x_{N_{1}+N_{2}: n}$ construct a simulated random sample from the MOGE distribution in stress level $s_{1}$, since using the probability integral transformation theorem, for $1 \leqslant j \leqslant N_{2}$,

$$
\begin{aligned}
& V_{j: m}=P\left(X \leqslant x_{N_{1}+j: n} \mid X \geqslant t_{1}\right)=\frac{G_{2}\left(x_{N_{1}+j: n}\right)-G_{1}\left(t_{1}\right)}{1-G_{1}\left(t_{1}\right)} \\
& \Longleftrightarrow V_{j: m}=\frac{1-e^{-\frac{x_{N_{1}+j: n}-t_{1}}{\theta_{2}}}}{1-(1-p) e^{-\frac{x_{N_{1}+j: n}-t_{1}}{\theta_{2}}-\frac{t_{1}}{\theta_{1}}}} \\
& x_{N_{1}+j: n}=t_{1}-\theta_{2} \log \frac{1-V_{j: m}}{1-V_{j: m}(1-p) e^{-\frac{t_{1}}{\theta_{1}}}} .
\end{aligned}
$$

Step 5. Based on $n, N_{1}, N_{2}, t_{1}, t_{2}$ and ordered observations $\left\{x_{1: n}, \ldots\right.$, $\left.x_{N_{1}: n}, x_{N_{1}+1: n}, \ldots, x_{N_{1}+N_{2}: n}\right\}$, we can obtain the MLEs $\left(\hat{p}, \hat{\theta_{1}}, \hat{\theta_{2}}\right)$ by solving system of nonlinear Equation (9), (12) and (13).

Step 6. Repeat Steps $1-5, r$ times representing $r$ different samples. The value of $r$ has been taken to be 1000 .

Step 7. If $\hat{\psi_{k l}}$ is a MLE of $\psi_{l}, l=1,2,3$ (where $\psi_{l}$ is a general notation that can be replaced by $p, \theta_{1}$ and $\theta_{2}$ i.e. $\psi_{1} \equiv p, \psi_{2} \equiv \theta_{1}$ and $\psi_{3} \equiv \theta_{2}$ ), based on sample $k, k=1, \ldots, r$ then the average estimate, MSE, RAB and $\mathrm{RE}$ of $\hat{\psi}_{l}$ over the $r$ samples are given, respectively by

$$
\begin{aligned}
& \overline{\hat{\psi}}_{l}=\frac{1}{r} \sum_{k=1}^{r} \hat{\psi_{k l}}, \\
& M S E\left(\hat{\psi}_{l}\right)=\frac{1}{r} \sum_{k=1}^{r}\left(\hat{\psi_{k l}}-\psi_{l}\right)^{2}, \\
& R A B\left(\hat{\psi}_{l}\right)=\frac{\left|\overline{\hat{\psi}_{l}}-\psi_{l}\right|}{\psi_{l}},
\end{aligned}
$$


$R E\left(\hat{\psi}_{l}\right)=\frac{\sqrt{M S E\left(\hat{\psi}_{l}\right)}}{\psi_{l}}$.

Step 8. From Step 7 compute $\overline{\hat{p}}, \overline{\hat{\theta}_{1}}, \overline{\hat{\theta_{2}}}, M S E(\hat{p}), M S E\left(\hat{\theta_{1}}\right), M S E\left(\hat{\theta_{2}}\right)$, $R A B(\hat{p}), R A B\left(\hat{\theta_{1}}\right), R A B\left(\hat{\theta_{2}}\right), R E(\hat{p}), R E\left(\hat{\theta_{1}}\right)$ and $R E\left(\hat{\theta_{2}}\right)$.

\section{Bootstrap Confidence Intervals}

In this section, we present several parametric bootstrap methods to construct CIs for $p, \theta_{1}$ and $\theta_{2}$, studentized-t interval, percentile interval, and adjusted percentile (BCa) interval; see Efron (1982) and Hall (1988) for more details.

The following steps are followed to obtain a bootstrap sample,

Step 1. Based on the original type-I censored sample, $\left\{x_{1: n}, \ldots\right.$, $\left.x_{N_{1}: n}, x_{N_{1}+1: n}, \ldots, x_{N_{1}+N_{2}: n}\right\}$, we obtain $\hat{p}, \hat{\theta}_{1}$ and $\hat{\theta}_{2}$.

Step 2. Based on $n$, we generate a random sample of size $n$ from Uniform $(0,1)$ distribution, and obtain the order statistics $\left(U_{1: n}, \ldots, U_{n: n}\right)$.

Step 3. For a given value of the stress change time $t_{1}$, Find $N_{1}^{*}$ such that

$$
U_{N_{1}^{*}: n}<\frac{e^{-\frac{t_{1}}{\hat{\theta}_{1}}}}{1-(1-\hat{p}) e^{-\frac{t_{1}}{\hat{\theta}_{1}}}} \leqslant U_{N_{1}^{*}+1: n} .
$$

For $1 \leqslant i \leqslant N_{1}^{*}$, we set

$$
x_{i: n}^{*}=\hat{\theta}_{1} \log \frac{1-(1-\hat{p}) U_{i: n}}{1-U_{i: n}} .
$$

Step 4. Next, we generate a random sample of size $m=n-N_{1}^{*}$ from Uniform $(0,1)$ distribution, and obtain the order statistics $\left(V_{1: m}, \ldots, V_{m: m}\right)$.

Step 5. For a given value of censoring time $t_{2}$, Find $N_{2}^{*}$ such that

$$
V_{N_{2}^{*}: m}<\frac{1-e^{\frac{t_{2}-t_{1}}{\hat{\theta}_{2}}}}{1-(1-\hat{p}) e^{-\frac{t_{2}-t_{1}}{\hat{\theta}_{2}}-\frac{t_{1}}{\hat{\theta}_{1}}}} \leqslant V_{N_{2}^{*}+1: m} .
$$

(c) 2012, SRTC Iran 
For $1 \leqslant j \leqslant N_{2}^{*}$, we then set

$$
x_{N_{1}+j: n}^{*}=t_{1}-\hat{\theta}_{2} \log \frac{1-V_{j: m}}{1-V_{j: m}(1-\hat{p}) e^{-\frac{t_{1}}{\hat{\theta}_{1}}}} .
$$

Step 6. Based on $n, N_{1}^{*}, N_{2}^{*}, t_{1}, t_{2}$ and ordered observations $\left\{x_{1: n}^{*}, \ldots\right.$, $\left.x_{N_{1}: n}^{*}, x_{N_{1}+1: n}^{*}, \ldots, x_{N_{1}+N_{2}: n}^{*}\right\}$, we can obtain the bootstrap estimates $\left(\hat{p}^{*}, \hat{\theta}_{1}^{*}, \hat{\theta}_{2}^{*}\right)$ by solving system of nonlinear Equation (9), (12) and (13).

Step 7. Repeat Steps $2-6, B$ times and arrange all $\hat{p}^{*}, \hat{\theta_{1}^{*}}, \hat{\theta_{2}^{*}}$ in ascending to obtain the bootstrap sample $\left\{\hat{\psi}_{l}^{*[1]}, \hat{\psi}_{l}^{*[2]}, \ldots, \hat{\psi}_{l}^{*[B]}\right\}, l=1,2,3$, where $\hat{\psi}_{1}^{*} \equiv \hat{p}^{*}, \hat{\psi}_{2}^{*} \equiv \hat{\theta}_{1}^{*}, \hat{\psi}_{3}^{*} \equiv \hat{\theta}_{2}^{*}$.

\subsection{Studentized-t Interval}

First, find the order statistics $T_{l}^{*[1]}<T_{l}^{*[2]}<\cdots<T_{l}^{*[B]}$, where

$$
T_{l}^{*[j]}=\frac{\hat{\psi}_{l}^{*[j]}-\hat{\psi}_{l}}{\sqrt{\operatorname{var}\left(\hat{\psi}_{l}^{*[j]}\right)}}, \quad j=1, \ldots, B, \quad l=1,2,3
$$

where $\hat{\psi}_{1}=\hat{p}, \hat{\psi}_{2}=\hat{\theta}_{1}$ and $\hat{\psi}_{3}=\hat{\theta}_{2}$.

A two-sided 100(1- $\alpha) \%$ studentized-t bootstrap confidence interval (TBCI) for $\psi_{l}$ is

$$
\left(\hat{\psi}_{l}-T_{l}^{*[(1-\alpha / 2) B]} \sqrt{\operatorname{var}\left(\hat{\psi}_{l}\right)}, \hat{\psi}_{l}-T_{l}^{*[(\alpha / 2) B]} \sqrt{\operatorname{var}\left(\hat{\psi}_{l}\right)}\right)
$$

where $\operatorname{var}\left(\hat{\psi}_{l}\right)$ is estimated as the asymptotic variance, obtained from Section 5 .

\subsection{Percentile Bootstrap Confidence Interval (PBCI)}

A two-sided $100(1-\alpha) \%$ percentile bootstrap confidence interval for $\psi_{l}$ is

$$
\left(\hat{\psi}_{l}^{*[(\alpha / 2) B]}, \hat{\psi}_{l}^{*[(1-\alpha / 2) B]}\right), \quad l=1,2,3 .
$$




\subsection{Adjusted Percentile (BCa) Interval}

A two-sided 100(1- $\alpha) \%$ BCa bootstrap confidence interval for $\psi_{l}$ is

$$
\left(\hat{\psi}_{l}^{*\left[\alpha_{1 l} B\right]}, \hat{\psi}_{l}^{*\left[\left(1-\alpha_{2 l}\right) B\right]}\right), \quad l=1,2,3 .
$$

where

$$
\alpha_{1 l}=\Phi\left(\hat{z}_{0 l}+\frac{\hat{z}_{0 l}+z_{\alpha / 2}}{1-\hat{a}_{l}\left(\hat{z}_{0 l}+z_{\alpha / 2}\right)}\right) \text {, }
$$

and

$$
\alpha_{2 l}=\Phi\left(\hat{z}_{0 l}+\frac{\hat{z}_{0 l}+z_{1-\alpha / 2}}{1-\hat{a}_{l}\left(\hat{z}_{0 l}+z_{1-\alpha / 2}\right)}\right) .
$$

Here, $\Phi(\cdot)$ is the CDF of the standard normal distribution. The value of the bias correction $\hat{z}_{0 l}$ can be computed as

$$
\hat{z}_{0 l}=\Phi^{-1}\left(\frac{\hat{\psi}_{l}^{*[j]}<\hat{\psi}_{l}}{B}\right), \quad j=1, \ldots, B, \quad l=1,2,3,
$$

while the acceleration $a_{l}$ is estimated by

$$
\hat{a}_{l}=\frac{\sum_{i=1}^{N_{1}+N_{2}}\left(\hat{\psi}_{l}^{(.)}-\hat{\psi}_{l}^{(i)}\right)^{3}}{6\left(\sum_{i=1}^{N_{1}+N_{2}}\left(\hat{\psi}_{l}^{(\cdot)}-\hat{\psi}_{l}^{(i)}\right)^{2}\right)^{3 / 2}}, \quad l=1,2,3,
$$

where $\hat{\psi}_{l}^{(i)}$ is the MLE of $\psi_{l}$ based on the simulated Type-I censored sample with the $i$ th observation deleted (i.e., the jackknife estimate), and

$$
\hat{\psi}_{l}^{(\cdot)}=\frac{1}{N_{1}+N_{2}} \sum_{i=1}^{N_{1}+N_{2}} \hat{\psi}_{l}^{(i)}, \quad l=1,2,3 .
$$

\section{Numerical Results}

All simulation results are summarized in Tables 1-3, based on 1000 simulations. Tables 1-3 show the average MLEs, MSEs, RABs and REs of $p, \theta_{1}$ and $\theta_{2}$ for different values of $t_{1}$ and $t_{2}$. The values of the population parameters are arbitrary chosen to be $p=0.5, \theta_{1}=12.18$ and $\theta_{2}=4.48$. The results are 
based on $n=35$ in Table $1, n=50$ in Table 2 and $n=100$ in Table 3 . The values of $t_{2}$ range from 7 to 10 by Step 1 and the values of $t_{1}$ are 6,7 and 8 .

Table 1. Average estimate of $p, \theta_{1}$ and $\theta_{2}$ with their MSE, RAB and RE for different stress change times and different censoring values based on 1000 simulations.

Population parameter values: $p=0.5, \theta_{1}=12.18, \theta_{2}=4.48$ with $n=35$.

\begin{tabular}{|c|c|c|c|c|c|c|}
\hline$t_{1}$ & $t_{2}$ & $\begin{array}{l}\bar{N}_{1} \\
\bar{N}_{2}\end{array}$ & $\begin{array}{l}\overline{\hat{\hat{p}}} \\
\overline{\hat{\theta_{1}}} \\
\overline{\hat{\theta_{2}}}\end{array}$ & $\begin{array}{l}\operatorname{MSE}(\hat{p}) \\
\operatorname{MSE}\left(\hat{\theta_{1}}\right) \\
\operatorname{MSE}\left(\hat{\theta_{2}}\right)\end{array}$ & $\begin{array}{l}\operatorname{RAB}(\hat{p}) \\
\operatorname{RAB}\left(\hat{\theta_{1}}\right) \\
\operatorname{RAB}\left(\hat{\theta_{2}}\right)\end{array}$ & $\begin{array}{l}\operatorname{RE}(\hat{p}) \\
\operatorname{RE}\left(\hat{\theta_{1}}\right) \\
\operatorname{RE}\left(\hat{\theta_{2}}\right)\end{array}$ \\
\hline \multirow[t]{4}{*}{6} & 7 & $\begin{array}{c}19.721 \\
4\end{array}$ & $\begin{array}{c}0.553 \\
36.402 \\
14.170\end{array}$ & $\begin{array}{c}0.170 \\
2153.315 \\
424.729\end{array}$ & $\begin{array}{l}0.106 \\
1.989 \\
2.163\end{array}$ & $\begin{array}{l}0.825 \\
3.810 \\
4.600\end{array}$ \\
\hline & 8 & $\begin{array}{c}19.521 \\
6.924\end{array}$ & $\begin{array}{c}0.551 \\
35.362 \\
11.337\end{array}$ & $\begin{array}{c}0.164 \\
2052.228 \\
234.32\end{array}$ & $\begin{array}{l}0.103 \\
1.903 \\
1.531\end{array}$ & $\begin{array}{l}0.810 \\
3.719 \\
3.417\end{array}$ \\
\hline & 9 & $\begin{array}{c}19.702 \\
8.82\end{array}$ & $\begin{array}{c}0.568 \\
32.992 \\
9.875\end{array}$ & $\begin{array}{c}0.165 \\
1853.139 \\
155.086\end{array}$ & $\begin{array}{l}0.135 \\
1.709 \\
1.204\end{array}$ & $\begin{array}{l}0.811 \\
3.534 \\
2.780\end{array}$ \\
\hline & 10 & $\begin{array}{l}19.671 \\
10.442\end{array}$ & $\begin{array}{c}0.587 \\
29.183 \\
8.191\end{array}$ & $\begin{array}{c}0.157 \\
1491.811 \\
90.181\end{array}$ & $\begin{array}{l}0.174 \\
1.396 \\
0.828\end{array}$ & $\begin{array}{l}0.793 \\
3.171 \\
2.120\end{array}$ \\
\hline \multirow[t]{3}{*}{7} & 8 & $\begin{array}{c}21.274 \\
3.662\end{array}$ & $\begin{array}{c}0.541 \\
35.447 \\
13.341\end{array}$ & $\begin{array}{c}0.161 \\
2042.395 \\
428.105\end{array}$ & $\begin{array}{l}0.082 \\
1.910 \\
1.978\end{array}$ & $\begin{array}{l}0.801 \\
3.710 \\
4.618\end{array}$ \\
\hline & 9 & $\begin{array}{l}21.26 \\
6.061\end{array}$ & $\begin{array}{c}0.559 \\
33.980 \\
10.799\end{array}$ & $\begin{array}{c}0.167 \\
1903.377 \\
210.932\end{array}$ & $\begin{array}{l}0.117 \\
1.790 \\
1.411\end{array}$ & $\begin{array}{l}0.818 \\
3.582 \\
3.242\end{array}$ \\
\hline & 10 & $\begin{array}{c}21.345 \\
7.798\end{array}$ & $\begin{array}{c}0.563 \\
31.715 \\
9.546\end{array}$ & $\begin{array}{c}0.156 \\
1717.846 \\
149.380\end{array}$ & $\begin{array}{l}0.127 \\
1.604 \\
1.131\end{array}$ & $\begin{array}{l}0.791 \\
3.403 \\
2.728\end{array}$ \\
\hline \multirow[t]{2}{*}{8} & 9 & $\begin{array}{c}22.703 \\
3.255\end{array}$ & $\begin{array}{c}0.557 \\
32.566 \\
11.313\end{array}$ & $\begin{array}{c}0.156 \\
1796.857 \\
257.157\end{array}$ & $\begin{array}{l}0.113 \\
1.674 \\
1.525\end{array}$ & $\begin{array}{l}0.790 \\
3.480 \\
3.579\end{array}$ \\
\hline & 10 & $\begin{array}{c}22.814 \\
5.234\end{array}$ & $\begin{array}{c}0.552 \\
32.753 \\
11.112\end{array}$ & $\begin{array}{c}0.156 \\
1806.050 \\
249.385\end{array}$ & $\begin{array}{l}0.104 \\
1.689 \\
1.480\end{array}$ & $\begin{array}{l}0.790 \\
3.489 \\
3.525\end{array}$ \\
\hline
\end{tabular}


Table 2. Average estimate of $p, \theta_{1}$ and $\theta_{2}$ with their MSE, RAB and RE for different stress change times and different censoring values based on 1000 simulations.

Population parameter values: $p=0.5, \theta_{1}=12.18, \theta_{2}=4.48$ with $n=50$.

\begin{tabular}{|c|c|c|c|c|c|c|}
\hline$t_{1}$ & $t_{2}$ & $\begin{array}{l}\bar{N}_{1} \\
\bar{N}_{2}\end{array}$ & $\begin{array}{l}\overline{\hat{\hat{p}}} \\
\overline{\hat{\theta_{1}}} \\
\overline{\hat{\theta_{2}}}\end{array}$ & $\begin{array}{l}\operatorname{MSE}(\hat{p}) \\
\operatorname{MSE}\left(\hat{\theta_{1}}\right) \\
\operatorname{MSE}\left(\hat{\theta_{2}}\right)\end{array}$ & $\begin{array}{l}\operatorname{RAB}(\hat{p}) \\
\operatorname{RAB}\left(\hat{\theta_{1}}\right) \\
\operatorname{RAB}\left(\hat{\theta_{2}}\right)\end{array}$ & $\begin{array}{l}\operatorname{RE}(\hat{p}) \\
\operatorname{RE}\left(\hat{\theta_{1}}\right) \\
\operatorname{RE}\left(\hat{\theta_{2}}\right)\end{array}$ \\
\hline 6 & 7 & $\begin{array}{c}28.059 \\
5.771\end{array}$ & $\begin{array}{c}0.534 \\
37.957 \\
13.380\end{array}$ & $\begin{array}{c}0.166 \\
2308.284 \\
339.406\end{array}$ & $\begin{array}{l}0.068 \\
2.116 \\
1.987\end{array}$ & $\begin{array}{l}0.814 \\
3.945 \\
4.112\end{array}$ \\
\hline & 8 & $\begin{array}{c}27.944 \\
9.872\end{array}$ & $\begin{array}{c}0.559 \\
33.437 \\
9.975\end{array}$ & $\begin{array}{c}0.159 \\
1878.894 \\
145.872\end{array}$ & $\begin{array}{l}0.118 \\
1.745 \\
1.227\end{array}$ & $\begin{array}{l}0.797 \\
3.559 \\
2.696\end{array}$ \\
\hline & 9 & $\begin{array}{l}28.060 \\
12.713\end{array}$ & $\begin{array}{c}0.560 \\
31.928 \\
9.329\end{array}$ & $\begin{array}{c}0.157 \\
1733.627 \\
122.167\end{array}$ & $\begin{array}{l}0.121 \\
1.621 \\
1.082\end{array}$ & $\begin{array}{l}0.793 \\
3.418 \\
2.467\end{array}$ \\
\hline & 10 & $\begin{array}{l}28.270 \\
14.765\end{array}$ & $\begin{array}{c}0.593 \\
26.349 \\
7.656\end{array}$ & $\begin{array}{c}0.147 \\
1250.328 \\
73.038\end{array}$ & $\begin{array}{l}0.186 \\
1.163 \\
0.709\end{array}$ & $\begin{array}{l}0.767 \\
2.903 \\
1.908\end{array}$ \\
\hline 7 & 8 & $\begin{array}{c}30.479 \\
5.057\end{array}$ & $\begin{array}{c}0.528 \\
33.322 \\
12.065\end{array}$ & $\begin{array}{c}0.148 \\
1807.009 \\
287.003\end{array}$ & $\begin{array}{l}0.055 \\
1.736 \\
1.693\end{array}$ & $\begin{array}{l}0.770 \\
3.490 \\
3.782\end{array}$ \\
\hline & 9 & $\begin{array}{c}30.407 \\
8.659\end{array}$ & $\begin{array}{c}0.552 \\
31.491 \\
9.675\end{array}$ & $\begin{array}{c}0.150 \\
1688.338 \\
148.085\end{array}$ & $\begin{array}{l}0.105 \\
1.585 \\
1.160\end{array}$ & $\begin{array}{l}0.774 \\
3.374 \\
2.716\end{array}$ \\
\hline & 10 & $\begin{array}{l}30.444 \\
11.187\end{array}$ & $\begin{array}{c}0.550 \\
29.962 \\
8.687\end{array}$ & $\begin{array}{c}0.146 \\
1513.144 \\
97.935\end{array}$ & $\begin{array}{l}0.101 \\
1.460 \\
0.939\end{array}$ & $\begin{array}{l}0.765 \\
3.194 \\
2.209\end{array}$ \\
\hline 8 & 9 & $\begin{array}{c}32.347 \\
4.437\end{array}$ & $\begin{array}{c}0.541 \\
31.857 \\
11.047\end{array}$ & $\begin{array}{c}0.146 \\
1682.816 \\
207.579\end{array}$ & $\begin{array}{l}0.082 \\
1.616 \\
1.466\end{array}$ & $\begin{array}{l}0.764 \\
3.368 \\
3.216\end{array}$ \\
\hline & 10 & $\begin{array}{c}32.453 \\
7.721\end{array}$ & $\begin{array}{c}0.543 \\
30.578 \\
9.113\end{array}$ & $\begin{array}{c}0.144 \\
1571.722 \\
122.445\end{array}$ & $\begin{array}{l}0.086 \\
1.510 \\
1.034\end{array}$ & $\begin{array}{l}0.758 \\
3.255 \\
2.470\end{array}$ \\
\hline
\end{tabular}


Table 3. Average estimate of $p, \theta_{1}$ and $\theta_{2}$ with their MSE, RAB and RE for different stress change times and different censoring values based on 1000 simulations.

Population parameter values: $p=0.5, \theta_{1}=12.18, \theta_{2}=4.48$ with $n=100$.

\begin{tabular}{|c|c|c|c|c|c|c|}
\hline$t_{1}$ & $t_{2}$ & $\begin{array}{l}\bar{N}_{1} \\
\bar{N}_{2}\end{array}$ & $\begin{array}{l}\overline{\hat{\hat{p}}} \\
\overline{\hat{\theta_{1}}} \\
\overline{\hat{\theta_{2}}}\end{array}$ & $\begin{array}{l}\operatorname{MSE}(\hat{p}) \\
\operatorname{MSE}\left(\hat{\theta_{1}}\right) \\
\operatorname{MSE}\left(\hat{\theta_{2}}\right)\end{array}$ & $\begin{array}{l}\operatorname{RAB}(\hat{p}) \\
\operatorname{RAB}\left(\hat{\theta_{1}}\right) \\
\operatorname{RAB}\left(\hat{\theta_{2}}\right)\end{array}$ & $\begin{array}{l}\operatorname{RE}(\hat{p}) \\
\operatorname{RE}\left(\hat{\theta_{1}}\right) \\
\operatorname{RE}\left(\hat{\theta_{2}}\right)\end{array}$ \\
\hline \multirow[t]{4}{*}{6} & 7 & $\begin{array}{l}56.068 \\
11.521\end{array}$ & $\begin{array}{c}0.554 \\
29.047 \\
9.556\end{array}$ & $\begin{array}{c}0.139 \\
1432.920 \\
130.639\end{array}$ & $\begin{array}{l}0.107 \\
1.385 \\
1.133\end{array}$ & $\begin{array}{l}0.747 \\
3.108 \\
2.551\end{array}$ \\
\hline & 8 & $\begin{array}{l}56.008 \\
19.806\end{array}$ & $\begin{array}{c}0.553 \\
28.445 \\
8.466\end{array}$ & $\begin{array}{c}0.137 \\
1360.074 \\
87.415\end{array}$ & $\begin{array}{l}0.105 \\
1.335 \\
0.890\end{array}$ & $\begin{array}{l}0.740 \\
3.028 \\
2.087\end{array}$ \\
\hline & 9 & $\begin{array}{l}55.683 \\
25.542\end{array}$ & $\begin{array}{c}0.525 \\
27.474 \\
8.014\end{array}$ & $\begin{array}{c}0.123 \\
1206.766 \\
70.589\end{array}$ & $\begin{array}{l}0.050 \\
1.256 \\
0.789\end{array}$ & $\begin{array}{l}0.702 \\
2.852 \\
1.875\end{array}$ \\
\hline & 10 & $\begin{array}{l}56.232 \\
29.601\end{array}$ & $\begin{array}{c}0.564 \\
24.083 \\
6.992\end{array}$ & $\begin{array}{c}0.126 \\
962.639 \\
45.530\end{array}$ & $\begin{array}{l}0.127 \\
0.977 \\
0.561\end{array}$ & $\begin{array}{l}0.709 \\
2.547 \\
1.506\end{array}$ \\
\hline \multirow[t]{3}{*}{7} & 8 & $\begin{array}{l}60.682 \\
10.211\end{array}$ & $\begin{array}{c}0.529 \\
27.969 \\
8.865\end{array}$ & $\begin{array}{c}0.125 \\
1289.749 \\
104.429\end{array}$ & $\begin{array}{l}0.058 \\
1.296 \\
0.979\end{array}$ & $\begin{array}{l}0.707 \\
2.949 \\
2.281\end{array}$ \\
\hline & 9 & $\begin{array}{l}60.692 \\
17.224\end{array}$ & $\begin{array}{c}0.543 \\
25.289 \\
7.760\end{array}$ & $\begin{array}{c}0.121 \\
1050.936 \\
68.393\end{array}$ & $\begin{array}{l}0.086 \\
1.076 \\
0.732\end{array}$ & $\begin{array}{l}0.695 \\
2.662 \\
1.846\end{array}$ \\
\hline & 10 & $\begin{array}{l}60.878 \\
22.327\end{array}$ & $\begin{array}{c}0.534 \\
24.017 \\
7.043\end{array}$ & $\begin{array}{c}0.113 \\
913.168 \\
43.645\end{array}$ & $\begin{array}{l}0.069 \\
0.972 \\
0.572\end{array}$ & $\begin{array}{l}0.672 \\
2.481 \\
1.475\end{array}$ \\
\hline 8 & 9 & $\begin{array}{c}65.057 \\
8.767\end{array}$ & $\begin{array}{c}0.516 \\
25.703 \\
8.591\end{array}$ & $\begin{array}{c}0.109 \\
1070.588 \\
92.071\end{array}$ & $\begin{array}{l}0.031 \\
1.110 \\
0.918\end{array}$ & $\begin{array}{l}0.662 \\
2.686 \\
2.142\end{array}$ \\
\hline & 10 & $\begin{array}{l}65.103 \\
15.157\end{array}$ & $\begin{array}{c}0.573 \\
22.368 \\
6.979\end{array}$ & $\begin{array}{c}0.119 \\
826.355 \\
49.926\end{array}$ & $\begin{array}{l}0.147 \\
0.836 \\
0.558\end{array}$ & $\begin{array}{l}0.689 \\
2.360 \\
1.577\end{array}$ \\
\hline
\end{tabular}

\subsection{Real Data}

In this section, we fit the MOGE model to one real data set. Data set is given by Gupta and Kundu (2003) on the failure times of the air conditioning 
system of the air plane 7912 . The data set is as follows:

$1,3,5,7,11,11,11,12,14,14,14,16,16,20,21,23,42,47,52,62,71,71,87,90$,

95, 120, 120, 225, 246, 261.

Here we fit the MOGE model to the real data set and show that the MOGE distribution is more flexible for analyzing of the data than the exponentiated exponential distribution (EE) and exponential distribution (E).

In order to compare the models, we usually used two criteria: Akaike Information Criterion (AIC) and BIC (Bayesian Information Criterion) which are defined as follows:

$$
A I C=-2 \log \hat{L}+2 k, \quad B I C=-2 \log \hat{L}+k \log (n),
$$

where $k$ is the number of free parameters in the model and $n$ is the sample size. For fitting a data set, the best model is a model with the smallest value of AIC and BIC statistics.

We can also perform formal goodness-of-fit tests in order to verify which distribution fits better to these data. We apply Kolmogorov-Smirnov (K$\mathrm{S})$ statistics and the p-value from the chi-square goodness of fit test, where the lower values of K-S statistic and the upper value of p-value for models indicate that these models could be chosen as the best model to fit the data.

The K-S statistic and the corresponding p-value evaluations were implemented using the $\mathrm{R}$ software through the command ks.test. MLEs of the model parameters and the values of the K-S statistics, P-value, AIC and BIC statistics are listed in Table 4.

Table 4. MLEs of the model parameters for Gupta and Kundu data, $\mathrm{K}-\mathrm{S}$ statistics, P-value and the measures AIC and BIC.

\begin{tabular}{cccccc}
\hline \hline Model & Parameters & K-S & P-value & AIC & BIC \\
\hline \multirow{2}{*}{ NGE } & $\hat{\theta}=0.10$ & 0.1268 & 0.7207 & 306.841 & 309.644 \\
& $\hat{p}=0.389$ & & & & \\
EE & $\hat{\alpha}=0.810$ & 0.1719 & 0.3382 & 308.401 & 311.204 \\
& $\hat{\lambda}=0.014$ & & & & \\
E & $\hat{\theta}=0.017$ & 0.2129 & 0.1319 & 307.2594 & 308.661 \\
\hline
\end{tabular}


Hence, it is clear that the new generalized exponential distribution fits quite well to this data set and is better than the exponentiated exponential distribution with the parameters $\alpha$ and $\lambda$ and the exponential distribution with the parameter $\theta$.

After analyzing the data, we found that the failure distribution of the air-conditioning system for the air plane is well by the new generalized exponential distribution. Now, we suppose this data of size $n=30$ and simple step-stress model under type-I censoring with $t_{1}=35$ and $t_{2}=90$ had occurred on this data. We then computed the MLEs of $p, \theta_{1}$ and $\theta_{2}$ and the estimates of their standard deviations. The MLE of parameters $p, \theta_{1}$ and $\theta_{2}$ are obtained as $\hat{p}=0.702, \hat{\theta_{1}}=56.003$ and $\hat{\theta_{2}}=81.909$. Also, standard deviations $p, \theta_{1}$ and $\theta_{2}$ are $0.985,62.689$ and 58.044, respectively.

\subsection{Illustrative Example}

In this subsection, we present an example to illustrate the estimation procedure, the asymptotic confidence interval and bootstrap CI methods for the parameters $p, \theta_{1}$ and $\theta_{2}$. In this example, we simulate a sample of size $n=35$, using the algorithm presented in Section 5 , based on population parameter values $p=0.5, \theta_{1}=12.18, \theta_{2}=4.48$. The two stress levels used in the simulation are $\theta_{1}=12.18$ and $\theta_{2}=4.48$. The stress change time $t_{1}$ and the censoring time $t_{2}$ are chosen to be equal 7 and 9 , respectively. Under type-I censoring, the simulated failure time data are presented in Table 5 , while the MLE, MSE, RAB and RE of the parameters are presented in Table 6 . Using the asymptotic confidence intervals (ACI) and bootstrap CIs presented in Sections 4 and 7, Table 7 shows $90 \%, 95 \%$ and $99 \%$ ACIs and bootstrap CIs for the parameters $p, \theta_{1}$ and $\theta_{2}$.

It can be seen from the simulated data, presented in Table 5, that there are 21 and 5 failure times in the intervals $(0,7]$ and $(7,9]$, respectively. From the results of Table 6 , the confidence interval length at $1-\alpha=0.90$ is smaller than the confidence interval length at $1-\alpha=0.95$ and $1-\alpha=0.99$. Also, the confidence interval length at $1-\alpha=0.95$ is smaller than the confidence interval length at $1-\alpha=0.99$. 
Table 5. Simulated data.

\begin{tabular}{cc}
\hline \hline $\begin{array}{c}\text { Stress Level } \theta_{1}=12.18 \\
\text { Failure times in the interval }(0,7]\end{array}$ & $\begin{array}{c}\text { Stress Level } \theta_{2}=4.48 \\
\text { Failure times in the interval }(7,9]\end{array}$ \\
\hline $0.0534,0.6067,0.7266,0.7848,1.1430,1.1931$, & \\
$1.2286,1.6487,1.9272,2.0842,2.0949,2.3394$, & $7.3320,7.4011,7.8178,8.3481,8.7218$, \\
$2.5292,2.9790,4.0447,4.2743,4.8495,4.8899$, & \\
$5.5299,5.6333,6.6981$, & \\
\hline
\end{tabular}

Table 6 MLEs of $p, \theta_{1}$ and $\theta_{2}$ with their MSE, RAB and RE. Population parameter values: $p=0.5, \theta_{1}=12.18$ and $\theta_{2}=4.48$ with $n=35$.

\begin{tabular}{|c|c|c|c|c|c|c|}
\hline$t_{1}$ & $t_{2}$ & $\begin{array}{l}N_{1} \\
N_{2}\end{array}$ & $\begin{array}{l}\hat{p} \\
\hat{\theta_{1}} \\
\hat{\theta_{2}}\end{array}$ & $\begin{array}{c}\operatorname{MSE}(\hat{p}) \\
\operatorname{MSE}\left(\hat{\theta_{1}}\right) \\
\operatorname{MSE}\left(\hat{\theta_{2}}\right)\end{array}$ & $\begin{array}{l}\operatorname{RAB}(\hat{p}) \\
\operatorname{RAB}\left(\hat{\theta_{1}}\right) \\
\operatorname{RAB}\left(\hat{\theta_{2}}\right)\end{array}$ & $\begin{array}{l}\operatorname{RE}(\hat{p}) \\
\operatorname{RE}\left(\hat{\theta_{1}}\right) \\
\operatorname{RE}\left(\hat{\theta_{2}}\right)\end{array}$ \\
\hline 7 & 9 & $\begin{array}{c}21 \\
5\end{array}$ & $\begin{array}{c}0.516 \\
12.142 \\
5.917\end{array}$ & $\begin{array}{c}0.0002 \\
0.001 \\
2.066\end{array}$ & $\begin{array}{l}0.032 \\
0.003 \\
0.321\end{array}$ & $\begin{array}{l}0.032 \\
0.003 \\
0.321\end{array}$ \\
\hline
\end{tabular}

Table 7. Asymptotic confidence intervals and bootstrap CIs of $p, \theta_{1}$ and $\theta_{2}$ based on $B=1000$ replications.

\begin{tabular}{ccccc}
\hline \hline parameters & Methods & $90 \%$ & $95 \%$ & $99 \%$ \\
\hline \multirow{2}{*}{$p$} & ACI & $(0,1)$ & $(0,1)$ & $(0,1)$ \\
& TBCI & $(0.0071,1)$ & $(0,1)$ & $(0,1)$ \\
& PBCI & $(0.0423,1)$ & $(0.038,1)$ & $(0.0307,1)$ \\
& BCa & $(0.0421,1)$ & $(0.0379,1)$ & $(0.0301,1)$ \\
\hline \multirow{6}{*}{$\theta_{1}$} & ACI & $(0,40.4356)$ & $(0,45.8559)$ & $(0,56.4494)$ \\
& TBCI & $(5.5222,43.0002)$ & $(4.4028,50.1454)$ & $(2.8538,69.9666)$ \\
& PBCI & $(5.7641,100)$ & $(5.4154,100)$ & $(4.4354,100)$ \\
& BCa & $(5.7126,100)$ & $(5.1652,100)$ & $(4.4109,100)$ \\
\hline \multirow{6}{*}{$\theta_{2}$} & ACI & $(0,15.4026)$ & $(0,17.2197)$ & $(0,20.7712)$ \\
& TBCI & $(2.6601,18.4992)$ & $(2.2923,23.9044)$ & $(1.8570,37.7206)$ \\
& PBCI & $(2.7852,50.2819)$ & $(2.3384,67.5619)$ & $(1.7659,100)$ \\
& BCa & $(1.8491,27.2998)$ & $(1.5657,35.5511)$ & $(0,58.2719)$ \\
\hline
\end{tabular}




\section{Conclusions}

In this paper, we considered a simple step-stress model with two stress levels from the MOGE distribution when there was time constraint on the duration of the experiment. We also obtained maximum likelihood equations for estimating the distribution parameters. In addition, asymptotic variance and covariance of the estimators were given. We also evaluated the properties of maximum likelihood estimation through the mean squared error, relative absolute bias and relative error. Furthermore, asymptotic confidence intervals of the estimators derived. We have also proposed several different procedures for constructing bootstrap confidence intervals. Finally, some simulation results are presented.

From results of Tables 1-3, we observe that:

1. For fixed values of $t_{1}$, by increasing $t_{2}$ the value of $\bar{N}_{2}$, the average of failures observed after $t_{1}$ before termination, increases and MSE and $\mathrm{RAB}$ of $\hat{\theta}_{1}$ and $\hat{\theta}_{2}$ decrease. The MSE of $\hat{p}$ decreases and RAB of $\hat{p}$ increases except for some cases. Also RE of $\hat{p}, \hat{\theta}_{1}$ and $\hat{\theta}_{2}$ decrease.

2. For fixed values of $t_{2}$, large values of $t_{1}$ would provide more data under low stress and less data under high stress, so the number of failures $\bar{N}_{1}$, the average number of failures before $t_{1}$, increases and $\bar{N}_{2}$ decreases. The MSE and RE of $\hat{p}$ decrease and also, RAB of $\hat{p}$ decreases except for some cases. The MSE, RAB and RE of $\hat{\theta}_{2}$ increase.

3. For fixed values of $t_{1}$ and $t_{2}$, as $n$ increases the MSE, RAB and RE of $\hat{\theta}_{1}$ and $\hat{\theta}_{2}$ decrease. Also, the MSE and RE of $\hat{p}$ decrease and the RAB of $\hat{p}$ decreases except for some cases.

\section{Acknowledgements}

Authors are grateful to the referees of the journal for their suggestions and for their help in writing the paper in acceptable form. 


\section{References}

Abdel-Hamid, A.H. and AL-Hussaini, E.K. (2009). Estimation in step-stress accelerated life tests for the exponentiated exponential distribution with Type-I censoring. Computational Statistics and Data Analysis, 53, 1328-1338.

Alice, T. and Jose, K.K. (1999). On Marshall- Olkin Generalized Exponential Distribution and its Applications. The Second Annual Conference of the Society of Statistics and Computer Applications held at St. Thomas College, Pala, Kerala during 28th November to 1st December 1999.

Bagdonavicius, V. (1978). Testing the hypothesis of additive accumulation of damages. Probability Theory and its Application, 23: 403-408.

Bagdonavicius, V. and Nikulin, M. (2002). Accelerated Life Models: Modeling and Statistical Analysis. Chapman Hall/CRC Press, Boca Raton, FL.

Bai, D.S., Kim, M.S. and Lee, S.H. (1989). Optimum simple step-stress accelerated life test with censoring. IEEE Transactions on Reliability, 38(5), 528-532.

Balakrishnan, N., Kundu, D., Ng, H.K.T. and Kannan, N. (2007). Point and interval estimation for a simple step-stress model with Type-II censoring. J. Qual Technol, 39, 35-47.

Balakrishnan, N. and Iliopoulos, G. (2009). Stochastic monotonicity of the MLEs of parameters in exponential simple step-stress models under Type-I and Type-II censoring. Metrika, 72, $89-109$.

Balakrishnan, N., Xie, Q. and Kundu, D. (2009). Exact inference for a simple step-stress model from the exponential distribution under time constraint. Ann Inst Stat Math, 61, 251-274.

Casella, G. and Berger, R.L. (2002). Statistical Inference, Second edition, Duxbury Press.

Chen, D.G. and Lio,Y.L. (2010) Parameter estimations for generalized exponential distribution under progressive type-I interval censoring. Computational Statistics and Data Analysis, 54(6), 1581-1591.

Cohen, A.C. (1965). Maximum likelihood estimation in the weibull distribution based on complete and on censored samples. Technometrics, 5, 579-588.

Efron, B. (1982). The Jackknife, the Bootstrap and Other Re-sampling Plans. In: CBMS/NSF Regional Conference Series in Applied Mathematics, Vol. 38. SIAM, Philadelphia, PA.

Gupta, R.D. and Kundu, D. (2003). Closeness of gamma and generalized exponential distribution, Communications in Statistics - Theory and Methods. 32, 705-721.

Hall, P. (1988). Theoretical comparison of bootstrap confidence intervals. Annals of Statistics, 16, 927-953. 
Harter, H.L. and Balakrishnan, N. (1996). CRC Handbook of Tables for the Use of Order Statistics in Estimation. CRC Press, Boca Raton, Florida.

Marshall, A.W. and Olkin, I. (1997). A new method for adding a parameter to a family of distributions with application to the exponential and Weibull families, Biometrika, 84, 641-652.

Meeker, W.Q. and Escobar, L.A. (1998). Statistical Methods for Reliability Data. Wiley, New York.

Miller, R. and Nelson, W. B. (1983). Optimum simple step-stress plans for accelerated life testing. IEEE Transactions on Reliability, 32, 59-65.

Nelson, W. (1980). Accelerated life testing: step-stress models and data analysis. IEEE Transactions on Reliability, 29, 103-108.

Nelson, W. (1990). Accelerated Testing: Statistical Models, Test Plans, and Data Analyses. Wiley, New York.

Sedyakin, N.M. (1966). On one physical priciple in reliability theory (in Russian). Techn. Cybernetics, 3, 80-87.

Sen, D. (1999). Accelerated Life Testing: Concepts and Models. Ph.D. Thesis, Concordia University, Canada.

Xiong, C. (1998). Inference on a simple step-stress model with Type-II censored exponential data. IEEE Trans. Reliab., 47, 142-146.

Xiong, C. and Milliken, G.A. (1999). Step-stress life-testing with random stress change times for exponential data. IEEE Trans. Reliab., 48, 141-148.

\section{F. L. Bagheri} Department of Statistics, Yazd University, Yazd, Iran.

\section{H. Torabi}

Department of Statistics, Yazd University, Yazd, Iran. email: htorabi@yazd.ac.ir 